DOI: $10.15593 / 2224-9982 / 2018.55 .06$

УДК 621.655.9: 629.7

\title{
Д.Ф. Хабарова, Е.К. Спиридонов
}

Южно-Уральский государственный университет

(национальный исследовательский университет), Челябинск, Россия

\section{ЭКСТРЕМАЛЬНЫЕ ХАРАКТЕРИСТИКИ И РАСЧЕТ БЕСКЛАПАННЫХ ПОРШНЕВЫХ НАСОСОВ С ГИДРАВЛИЧЕСКИМИ ДИОДАМИ}

\begin{abstract}
Статья посвящена насосам с вытеснителем возвратно-поступательного действия и гидравлическими диодами, которые ввиду отсутствия подвижных частей в проточной части нашли применение в системах повышенной ответственности в ядерной промышленности, а также в медицинской промышленности в системах транспорта лекарственных средств. Приведены аналитические выражения расходно-напорной характеристики и КПД поршневого насоса с гидравлическими диодами в размерных и безразмерных параметрах, уточненные численным исследованием энергетических характеристик насоса. В результате уточнения были получены корректирующие коэффрициенты $\varepsilon$ и $\psi$, а также установлено, что данные коэффициенты являются функцией диодности по сопротивлению гидравлических диодов. Функциональная зависимость представлена в графическом виде. На основе уточненных выражений рассчитаны и проанализированы экстремальные по КПД характеристики, которые представляют собой максимальные возможности насоса. Анализом этих характеристик установлено, что оптимальная относительная подача насоса, т.е. подача, выраженная в долях идеальной подачи герметичного насоса, зависит главным образом от диодности гидравлических диодов, при этом величина коэффициента гидравлического сопротивления в прямом направлении диода не оказывает сколь угодно существенного влияния на величину оптимальной относительной подачи. Экстремальные характеристики представле-

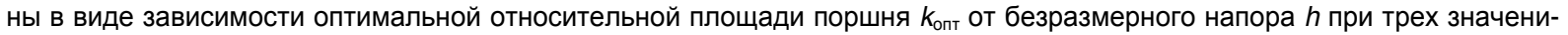

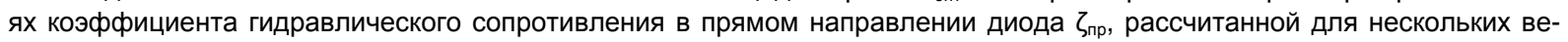
личин диодности $D$. Эти характеристики могут служить основой для расчета насоса при проектировании. Приведен числовой пример последовательности расчета насоса на заданные подачу $Q$ и напор $H$.
\end{abstract}

Ключевые слова: поршневой насос, бесклапанные насосы, гидравлические диоды, экстремальные характеристики, расчет, проектирование.

\section{D.F. Khabarova, E.K. Spiridonov}

South Ural State University (National Research University), Chelyabinsk, Russian Federation

\section{EXTREME CHARACTERISTICS AND CALCULATION OF A VALVELESS PISTON PUMP WITH FLUIDIC DIODES}

The article is focused on the pumps with a reciprocating displacer and fluidic diodes, which, due to the lack of moving parts in the wet part, are applied in safety-critical systems in the nuclear industry, as well as in the medical industry in drug transport systems. The article presents analytic expressions of the flow rate and head characteristics and efficiency of a piston pump with hydraulic diodes given in dimensional and dimensionless parameters, updated by numerical investigation of the energy characteristics of the pump. As a result of the update, correcting coefficients $\varepsilon$ and $\psi$ were obtained, and it was also revealed that these coefficients are functions of the diodicity of the fluidic diodes. The functional relations are presented in graphical form. Based on the updated expressions, the extreme characteristics, which represent the maximum achievable pump capabilities, were calculated and analyzed. By analyzing these characteristics, it was found that the optimal non-dimensional pump flow rate, i.e. the flow rate, expressed in fractions of the ideal flow of a absolute leakless pump, depends mainly on the diodicity of the fluidic diodes. Moreover, the value of the coefficient of hydraulic resistance in the forward direction of the diode does not have an arbitrarily significant influence on the value of the optimal non-dimensional flow rate. Extreme characteristics are represented in the form of the dependence of the optimum relative area of the piston $k_{\text {опт }}$ on the non-dimensional head pressure with three values of the hydraulic resistance coefficient in the forward direction of the diode $\zeta_{\text {пр }}$, calculated for several values of diodicity $D$. An example of the pump calculation sequence for certain flow rate $Q$ and head $H$ is given

Keywords: piston pump, valveless pumps, fluidic diodes, extreme characteristics, calculation, design. 


\section{Введение}

Насосы с вытеснителем возвратно-поступательного действия и гидравлическими диодами нашли применение в медицинской [1] и ядерной [2] промышленности вследствие их относительно высокой надежности и отсутствия подвижных частей в проточной части, которые могли бы разрушать чувствительную структуру перекачиваемых сред. Так, в медицинской промышленности для транспорта лекарственных средств применяются мембранные микронасосы одно- и двухстороннего действия. В качестве органов распределения в них чаще всего используются диоды типа диффузор-конфузор и диоды Тесла [3, 4]. В ядерной промышленности такие насосы представлены в рамках направления PowerFluidics $[2,5,6]$. Вытеснителем в них служит воздух, попеременно нагнетаемый и откачиваемый из насосной камеры, а распределение жидкости осуществляется с помощью вихревых диодов.

Существует целый ряд исследований некоторых конкретных конструкций такого типа насосов: мембранных микронасосов [3, 7, 4], насосов с газовым вытеснителем [8], а также насосов с поршневым вытеснителем [9-13]. Причем наиболее обширные исследования выполнены для микронасосов. Исследования последних двух видов конструкций сводились в основном к аналитическому анализу рабочего процесса и прогнозу энергетических характеристик. Несмотря на то, что насосы с вытеснителем возвратно-поступательного действия и гидравлическими диодами применяются уже десятки лет, общей теории проектирования и исследований потенциальных возможностей таких насосов обнаружить не удалось.

Глубокое изучение и разработка общей теории расчета и проектирования таких бесклапанных насосов позволят существенно расширить область их применения. Так, например, относительно высокая надежность делает их привлекательными для ответственных систем наземной инфраструктуры ракетно-космической техники, а также для различных гидропневмосистем авиации. Кроме того, благодаря возможности перекачивания многокомпонентных жидкостей и жидкостей с чувствительной структурой такие насосы могут найти применение в средствах заправки ракет и авиационной техники $[14,15]$.

Таким образом, исследование экстремальных характеристик поршневых насосов с гидравлическими диодами, которые служат основой для проектирования таких гидромашин, является актуальной задачей.

\section{Экстремальные характеристики}

Потенциальные возможности насосов отражаются их экстремальными характеристиками. Последние устанавливают взаимосвязь безразмерных геометрических и режимных параметров, при которых достигается наибольший КПД насоса.

В статье [11] приведена расчетная модель поршневого насоса с гидравлическими диодами, принципиальная схема которого показана на рис. 1. На основе этой модели авторы получают аналитические зависимости подачи и КПД такого насоса от размерных и безразмерных режимных и геометрических параметров. Дальнейшее исследование в этом направлении позволило уточнить расчетную модель путем ввода в нее корректирующих коэффициентов, полученных в результате численного эксперимента. С учетом корректирующих коэффициентов выражения подачи и КПД насоса имеют вид

$$
Q_{\mathrm{H}}=\frac{\pi d_{\mathrm{Tp}}^{2}}{4}\left(\frac{2 r \frac{d_{\mathrm{\pi}}^{2}}{d_{\mathrm{tp}}^{2}}(D+1)}{\varepsilon(D-1) T}-\psi \sqrt{\frac{g\left(H_{2}-H_{1}\right)}{\zeta_{\text {пр }} D}}\right),
$$




$$
\eta=\frac{\rho g\left(H_{2}-H_{1}\right) \frac{\pi d_{\mathrm{Tp}}^{2}}{2}\left(\frac{2 r \frac{d_{n}^{2}}{d_{\mathrm{Tp}}^{2}}(D+1)}{(D-1) \varepsilon}-\psi T \sqrt{\frac{g\left(H_{2}-H_{1}\right)}{\zeta_{\mathrm{mp}} D}}\right)}{\frac{\pi d_{\mathrm{\pi}}^{2} r}{4 \psi^{2}}\left(1+\frac{1}{D}\right) \rho g\left(H_{2}-H_{1}\right)+\frac{\pi \omega^{2} r^{3} \frac{d_{\mathrm{\pi}}^{4}}{d_{\mathrm{Tp}}^{2}}(D+1)}{(D-1)}\left(\frac{\rho \zeta_{\text {пр }} \frac{d_{\mathrm{\pi}}^{2}}{d_{\mathrm{\tau p}}^{2}} D}{3(D-1) \varepsilon}+\frac{\pi \rho \sqrt{g\left(H_{2}-H_{1}\right) \zeta_{\mathrm{mp}}}}{4 \omega r \psi}\right)},
$$

где $Q_{\text {н }}$ - подача насоса, м ${ }^{3} / \mathrm{c} ; \eta$ - гидравлический КПД насоса; $d_{\text {тр }}-$ диаметр подводящего и отводящего патрубков, м; $D, \zeta_{\text {пр }}$ диодность и коэффициент гидравлического сопротивления гидравлических диодов в прямом направлении; $T$ - период одного цикла работы насоса, с, $T=2 \pi / \omega ; \omega-$ угловая скорость вращения вала кривошипа, с; $r$ - радиус кривошипа, м; $H_{2}$ и $H_{1}-$ напоры в напорном и всасывающих успокоителях соответственно, м; $d_{\text {п }}-$ диаметр поршня насоса, м; $\varepsilon$ и $\psi$ - корректирующие коэффициенты.

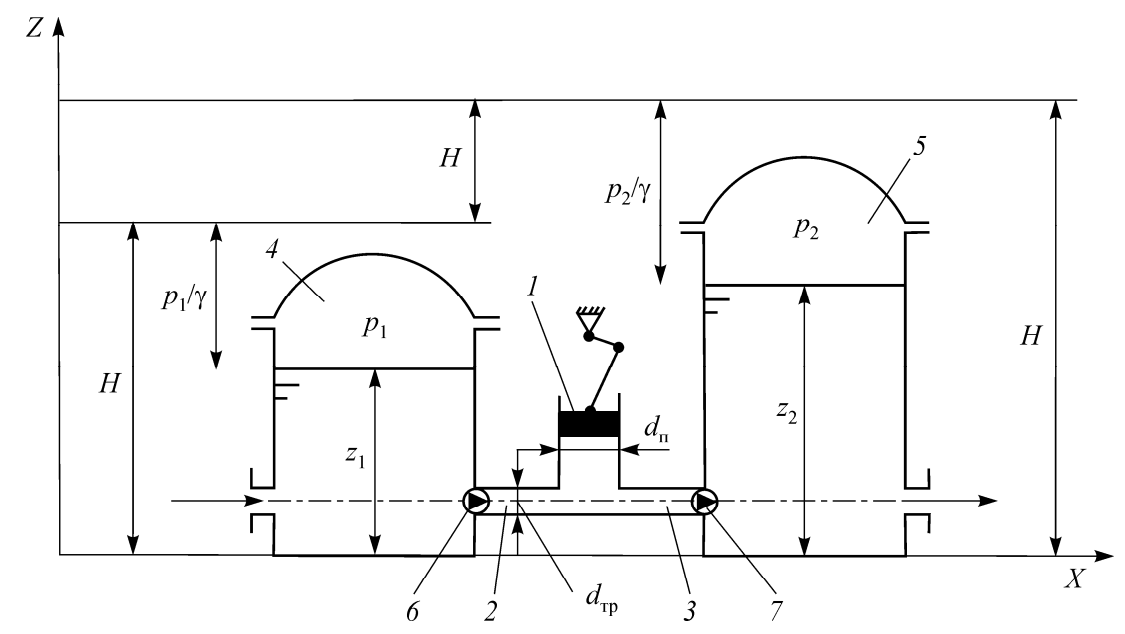

Рис. 1. Принципиальная схема бесклапанного поршневого насоса: 1 - поршень с кривошипно-шатунным приводом; 2, 3 - всасывающий и напорный патрубки; 4, 5 - всасывающий и напорный успокоители;

6, 7 - всасывающий и напорный гидравлические диоды

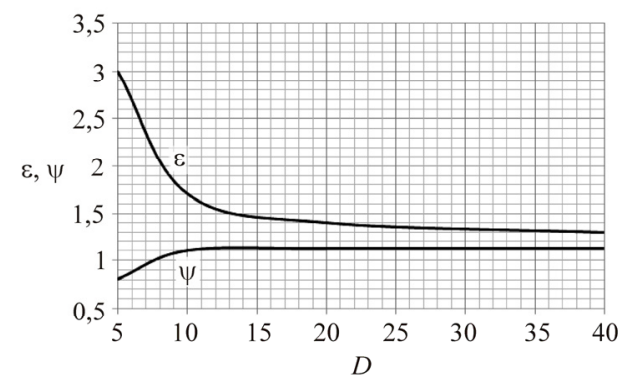

Рис. 2. Зависимость корректирующих коэффициентов от диодности гидравлических диодов

Исследованием было установлено, что корректирующие коэффициенты $\varepsilon$ и $\psi$ зависят главным образом от диодности гидравлических диодов, причем эта зависимость существенна только при $D<15$. На рис. 2 представлены графики функций коэффициентов $\varepsilon$ и $\psi$ от диодности гидродиодов $D$.

$\mathrm{C}$ целью упрощения применения расчетной модели для предварительных проектировочных расчетов введем безразмерные параметры [11]:

1) $q$ - относительная подача насоса:

$$
0 \leq q=\frac{Q_{\text {н }}}{Q_{\text {ид }}} \leq 1,
$$

где $Q_{\text {ид }}$ - идеальная подача насоса с клапанным распределением (без утечек), $\mathrm{m}^{3} / \mathrm{c}$, $Q_{\text {ид }}=\frac{W_{\text {цил }}}{T}=\frac{2 r \pi d_{\text {п }}^{2}}{4 T}$. Таким образом, 


$$
0 \leq q=\frac{4 Q_{\mathrm{H}}}{\omega r d_{\Pi}^{2}} \leq 1
$$

2) $k$ - относительная площадь поршня:

$$
k=\frac{d_{\text {п }}^{2}}{d_{\mathrm{Tp}}^{2}} ;
$$

3) $h$ - напор насоса в долях максимального скоростного напора под поршнем:

$$
h=\frac{H_{2}-H_{1}}{\frac{v_{\mathrm{n} \max }^{2}}{2 g}}=\frac{2 g\left(H_{2}-H_{1}\right)}{\omega^{2} r^{2}},
$$

где $v_{\text {п } \max }-$ максимальная скорость поршня, $v_{\text {п } \max }=\omega r$.

Преобразовав уравнение (1) с учетом введенных безразмерных параметров, получаем выражение расходно-напорной характеристики насоса в безразмерных координатах:

$$
q=\frac{D+1}{\varepsilon(D-1)}-\frac{\pi}{k} \psi \sqrt{\frac{h}{2 \zeta_{\text {пр }} D}}
$$

Введем в формулу (2) безразмерные параметры, после преобразования получим выражение

$$
\eta=\frac{4 h \frac{1}{k}\left(\frac{k(D+1)}{\varepsilon(D-1)}-\pi \psi \sqrt{\frac{h}{2 \zeta_{\text {пр }} D}}\right)}{\frac{h}{\psi^{2}}\left(1+\frac{1}{D}\right)+\frac{2 k(D+1)}{(D-1)}\left(\frac{4 \zeta_{\text {пр }} D k}{3(D-1) \varepsilon}+\frac{\pi}{2 \psi} \sqrt{\frac{h \zeta_{\text {пр }}}{2}}\right)} .
$$

Анализ уравнений (6) и (7) вместе с графиками на рис. 2 показывает, что КПД поршневого насоса одностороннего действия зависит в первую очередь от относительных напора $h$ и подачи $q$, относительной площади поршня $k$, диодности по сопротивлению гидродиодов $D$ и их гидравлического сопротивления при течении жидкости в прямом направлении $\zeta_{\text {пр. }}$.

Численный анализ безразмерных расходно-напорных [11-13] характеристик показывает, что на каждой из них имеется точка с наибольшим КПД. На рис. 3-6 представлены расчетные расходно-напорные характеристики (сплошные линии) и КПД (штриховые линии) насоса при различных диодностях и относительных площадях поршня $k$. Вертикальными и горизонтальными пунктирными линиями обозначены относительные значения напора и подачи, при которых достигается наибольший КПД. Например, при диодности гидравлических диодов $D=10$, гидравлическом сопротивлении в прямом направлении $\zeta_{\text {пр }}=2$ и относительной площади поршня $k=16$ максимальный КПД достигается при относительном напоре $h=158,6$ и относительной подаче $q=0,289$.

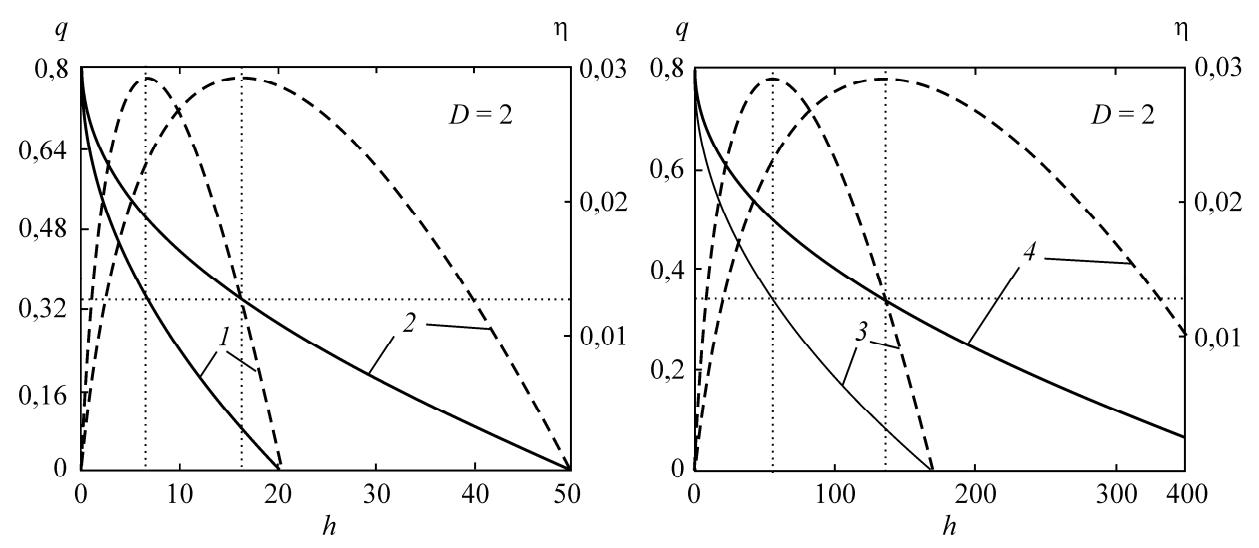

Рис. 3. Безразмерные расходно-напорные характеристики и КПД насоса при диодности $D=2, \zeta_{\text {пр }}=1: 1-k=4 ; 2-k=6,25 ; 3-k=16 ; 4-k=25$ 


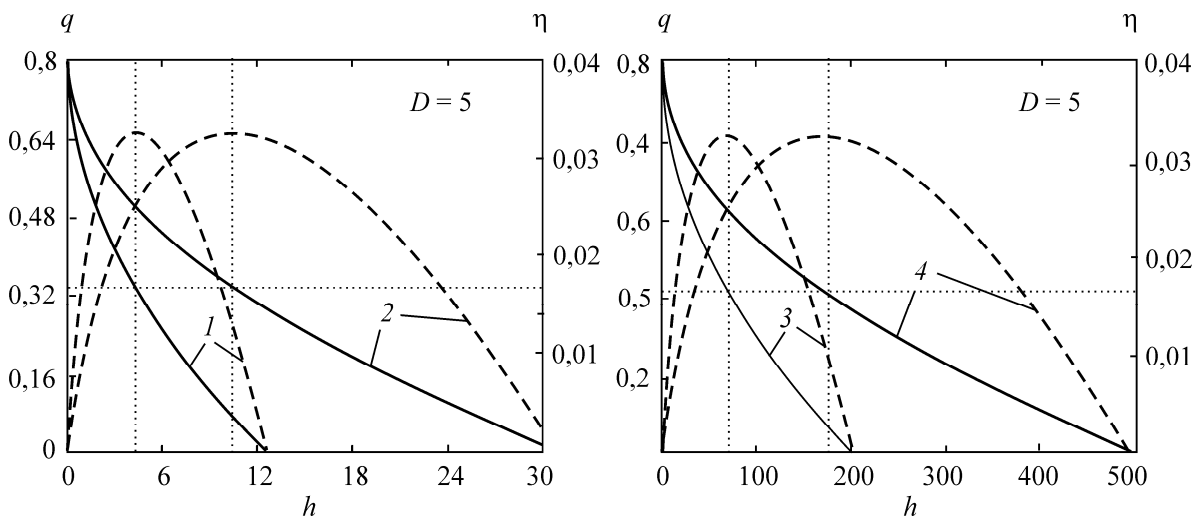

Рис. 4. Безразмерные расходно-напорные характеристики и КПД насоса при диодности $D=5, \zeta_{\text {пр }}=1: 1-k=4 ; 2-k=6,25 ; 3-k=16 ; 4-k=25$

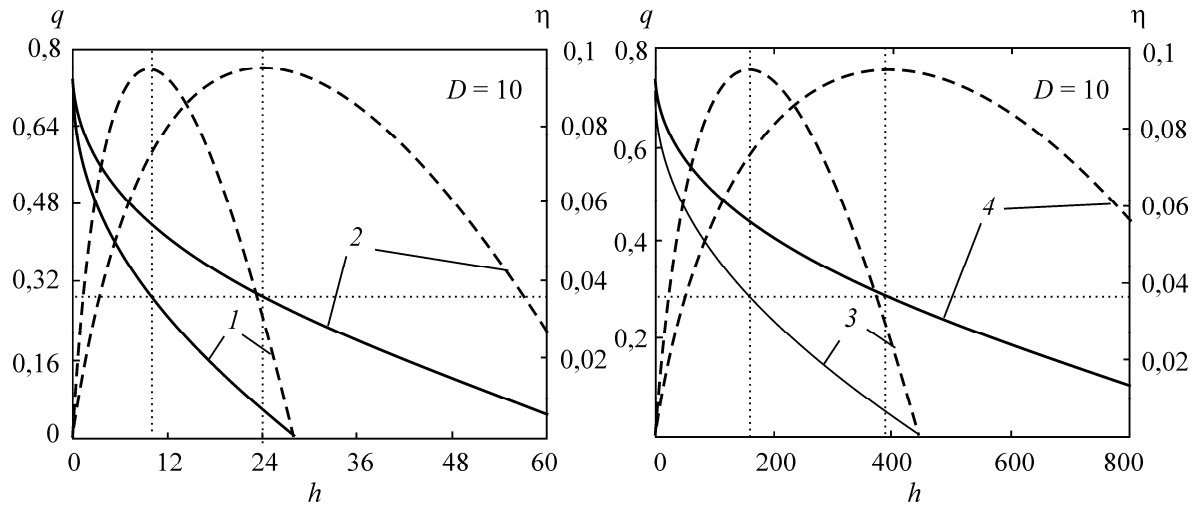

Рис. 5. Безразмерные расходно-напорные характеристики и КПД насоса при диодности $D=10, \zeta_{\text {пр }}=2: 1-k=4 ; 2-k=6,25 ; 3-k=16 ; 4-k=25$
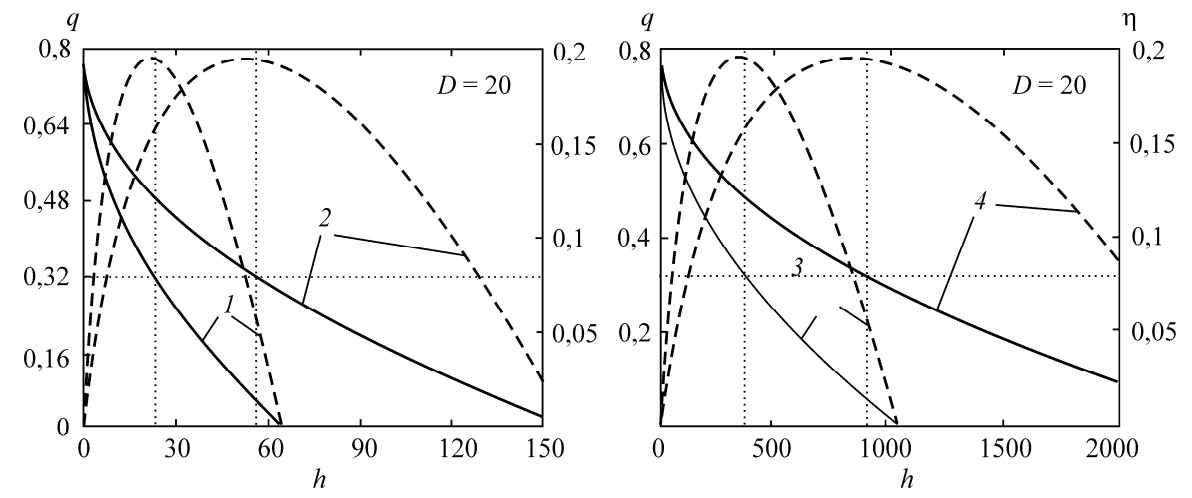

Рис. 6. Безразмерные расходно-напорные характеристики и КПД насоса при диодности $D=20, \zeta_{\text {пр }}=2: 1-k=4 ; 2-k=6,25 ; 3-k=16 ; 4-k=25$

Как видно по рисункам, оптимальная относительная подача насоса $q_{\text {опт }}$, т.е. подача, при которой достигается наибольший КПД, зависит только от диодности по сопротивлению гидравлических диодов. Эта зависимость представлена на рис. 7. 
Исследованием функции (7) КПД насоса от его относительного напора $h$ на экстремум при различных значениях относительной площади поршня $k$ и том или

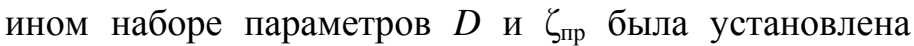
взаимосвязь между относительной площадью поршня и относительным напором насоса, при которых достигается максимальный КПД. Последняя проиллюстрирована на рис. 8-11. На каждом из этих рисунков показаны зависимости оптимальной относительной площади поршня от относительного напора насоса при определенном значении диодности гидравлических диодов и трех значениях прямого сопротивления $\zeta=1,2$ и 3, а также указана оптимальная относитель-

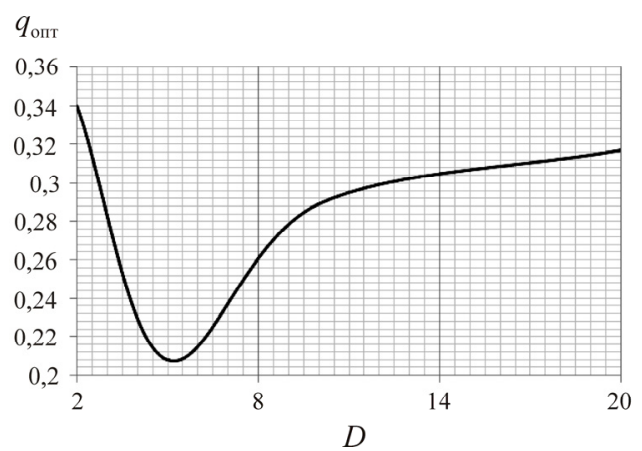

Рис. 7. Зависимость оптимальной подачи насоса от диодности по сопротивлению гидродиодов ная подача при данной диодности. Так, например, на рис. 8 показаны зависимости при $D=2$ и оптимальной относительной подаче $q_{\text {опт }}=0,34$.

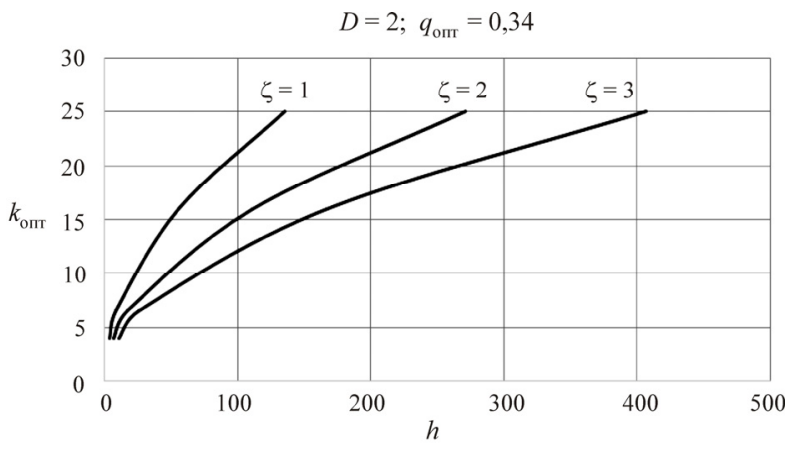

Рис. 8. Зависимость относительной площади поршня насоса от оптимального напора при диодности $D=2$

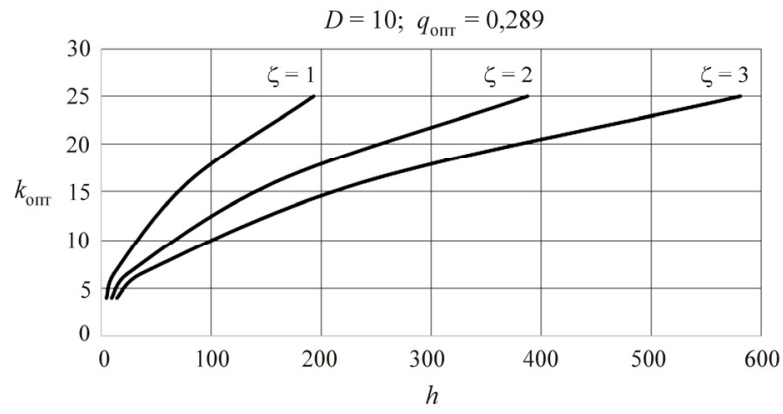

Рис. 10. Зависимость относительной площади поршня насоса от оптимального напора при диодности $D=10$

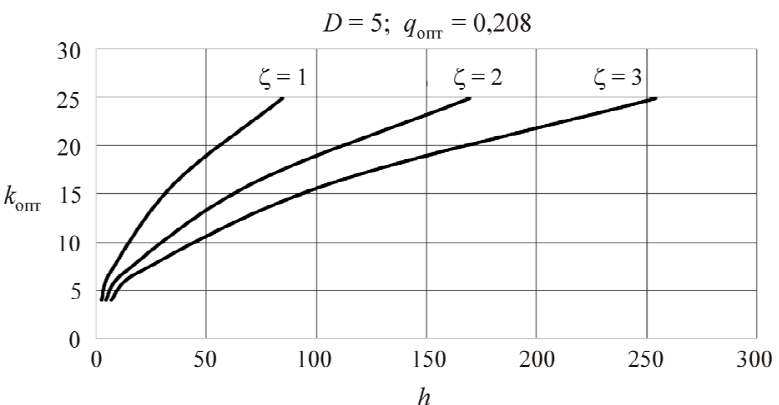

Рис. 9. Зависимость относительной площади поршня насоса от оптимального напора при диодности $D=5$

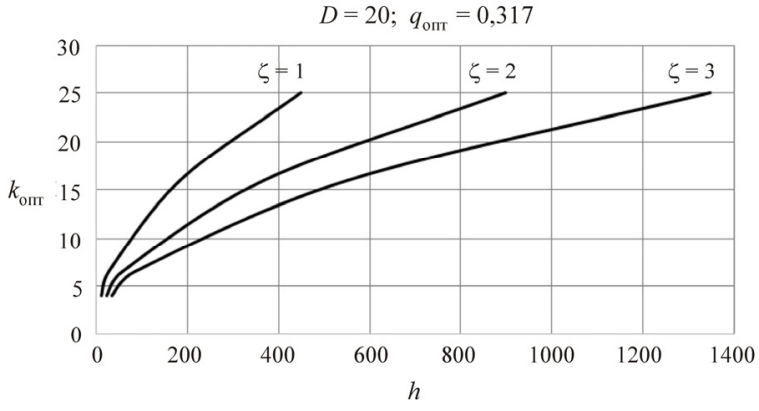

Рис. 11. Зависимость относительной площади поршня насоса от оптимального напора при диодности $D=20$

Кривые на рис. 8-11 отражают максимальные возможности насоса и служат основой для предварительного проектировочного расчета поршневого насоса с гидравлическими диодами.

\section{Расчет насоса}

Рассмотрим последовательность расчета при проектировании насоса с кривошипношатунным приводом поршня на заданные подачу $Q$ и напор $H_{\text {н }}$ на конкретном числовом примере. Исходными данными при расчете являются: подача насоса $Q=1$ л/с; напор $H=20$ м; параметры привода, а именно частота вращения вала кривошипа $n=1000$ об/мин (угловая ско- 
рость вращения $\omega=104,7$ рад/с) и его радиус $r=15$ мм; тип гидравлических диодов - диффузорные, их диодность $D=10$ и коэффициент гидравлического сопротивления при течении жидкости в прямом направлении $\zeta_{\text {пр }}=2$. При расчете все размеры переведем в единицы СИ.

Следует отметить, что тип гидравлического диода выбирается исходя из условий работы и области применения насоса. Так, например, вихревые диоды имеют сравнительно высокую диодность, но при этом обладают высокой инерционностью и ограничивают частоту вращения привода насоса, что, в свою очередь, ведет к увеличенным габаритам насоса. В целом, выбор типа гидравлических диодов является комплексной задачей и требует дополнительного исследования.

Параметры привода насоса также определяются в большей степени областью применения насоса. Кроме того, существуют некоторые конструктивные ограничения. Так, например, линейная скорость поршня, а следовательно, и частота вращения вала кривошипа ограничиваются выбором уплотнения поршня. Кроме того, при выборе частоты вращения следует учитывать возможные кавитационные явления в насосе.

Этапы расчета:

1) на основании известной диодности гидродиодов $D=10$ по графику на рис. 7 определяется оптимальная подача насоса $q_{\text {опт }}=0,289$;

2) из известных напора $H_{\mathrm{H}}=20$ м, угловой скорости $\omega=104,7$ рад/с и радиуса кривошипа $r=15$ мм по формуле (5) определяется безразмерный напор насоса $h$ :

$$
h=\frac{2 \cdot g \cdot 20}{104,7^{2} \cdot 0,015^{2}}=160 ;
$$

3) на графике экстремальных по КПД характеристик, соответствующем диодности гидродиодов $D=10$ (см. рис. 10), на основании рассчитанного безразмерного напора и заданного коэффициента прямого сопротивления $\zeta_{\text {пр }}=2$ гидродиода определяется оптимальная относительная площадь поршня $k_{\text {опт }}=16$;

4) по известным угловой скорости $\omega=104,7$ рад/с и радиусу кривошипа $r=15$ мм, а также оптимальной безразмерной подаче $q_{\text {опт }}=0,289$ из формулы (3) рассчитывается диаметр поршня насоса $d_{\text {п }}$ м:

$$
d_{\text {п }}=\sqrt{\frac{4 Q_{\mathrm{H}}}{\omega r q}}=\sqrt{\frac{4 \cdot 10^{-3}}{104,7 \cdot 0,015 \cdot 0,289}}=0,094 ;
$$

5) из формулы (4) по известным диаметру поршня $d_{\text {п }}=94$ мм и его оптимальной относительной площади рассчитывается диаметр подводящего и отводящего патрубков $d_{\text {тр }}$ м:

$$
d_{\text {тр }}=\sqrt{\frac{d_{\Pi}^{2}}{k}}=\sqrt{\frac{0,094^{2}}{16}}=0,024 .
$$

При этом следует отметить, что данный параметр не является диаметром магистрального трубопровода. Это диаметр патрубков, соединяющих поршневую камеру насоса и успокоители.

Таким образом, изложенный выше алгоритм расчета позволяет при эскизном проектировании насоса определить геометрические параметры его проточной части, а именно диаметры поршня и подводящих патрубков.

\section{Заключение}

На основе физико-математической модели рабочего процесса поршневого насоса с гидравлическими диодами[9] получены экстремальные по КПД характеристики, отражающие его максимальные возможности. Эти зависимости могут быть использованы для расчета насоса при его эскизном проектировании, а также послужить основой для разработки методики расчета и проектирования поршневых насосов с гидравлическими диодами. 


\section{Библиографический список}

1. Woias P. Micropumps - past, progress and future prospects // Sensors and Actuators. - 2005. No. 105. - P. 28-38.

2. NuVision Engineering, Inc.: [сайт]. - URL: http://www.nuvisioneng.com/ (accessed 25 November 2018).

3. Eladi P.B., Chatterjee D., DasGupta A. Design and development of a piezoelectrically actuated micropump for drug delivery application // Micro and Smart Devices and Systems. - Springer, 2014. P. 127-141.

4. Stemme E., Stemme G. A valveless diffuser/nozzle-based fluid pump // Sens. Actuators. - 1993. No. 39. - P. 159-167.

5. Etherington C. Power fluidics technology and its application in the nuclear industry // Nucl Energy. 1984. - Vol. 23(4). - P. 227-235.

6. Kletz T.A., Amyotte P. Process plants: a handbook for inherently safer design. - CRC Press, 2010. $224 \mathrm{p}$.

7. A valve-less diffuser micropump for microfluidic analytical systems / H. Andersson, W. van der Wijngaart, P. Nilsson, P. Enoksson, G. Stemme // Proceedings of the $\mu$ TAS 2000 Symposium. Springer, 2000 - P. 95-98.

8. Wada T., Takagi M., Shimizu A. Study on a fluidic pump with vortex diodes // Fluid Control and Measurement. - Tokyo: Pergamon Press, 1986. - P. 421-426.

9. Takagi S., Saijo T. Study of a piston pump without valves (1st report, on a pipe-capacity-system with a T-junction) // Bull. JSME - 1983. - No. 26. - P. 1366-1372.

10. Takagi S., Takahashi K. Study of a piston pump without valves (2st report, on a pipe-capacity-system with a T-junction) // Bull. JSME. - 1985. - Vol. 28, no. 239. - Р. 831-836.

11. Спиридонов Е.К., Хабарова Д.Ф. Расчетная модель и характеристики бесклапанного поршневого насоса одностороннего действия // Вестник ЮУрГУ. Машиностроение. - 2014. - Т. 14, № 4. - С. $13-22$.

12. Spiridonov E.K., Khabarova D.F. Computational model and characteristics of a double-acting valveless piston pump // Chemical and Petroleum Engineering. - Springer, 2016. - Vol. 52. - P. 539-546.

13. Спиридонов Е.К., Хабарова Д.Ф. Принципиальные схемы и характеристики бесклапанных насосов с вытеснителем возвратно-поступательного перемещения // Известия Самарского научного центра Российской академии наук. - 2016. - Т. 18, № 1(2). - С. 309-313.

14. Технологические объекты наземной инфраструктуры ракетно-космической техники: инж. пособие / под общ. ред. И.В Бармина. - М.: Полиграфикс РПК, 2005. - Кн. 1. - 416 с.

15. Технологические объекты наземной инфраструктуры ракетно-космической техники: инж. пособие / под общ. ред. И.В Бармина. - М.: Полиграфикс РПК, 2006. - Кн. 2. - 376 с.

\section{References}

1. Woias P. Micropumps - past, progress and future prospects. Sensors and Actuators, 2005, no. 105, pp. $28-38$.

2. NuVision Engineering, Inc. Available at: http://www.nuvisioneng.com/ (accessed 25 November 2018).

3. Eladi P.B., Chatterjee D., DasGupta A. Design and Development of a Piezoelectrically Actuated Micropump for Drug Delivery Application. Micro and Smart Devices and Systems, Springer, 2014, pp. 127-141.

4. Etherington C. Power fluidics technology and its application in the nuclear industry. Nucl Energy, 1984, vol. 23(4), pp. 227-235.

5. Kletz T.A., Amyotte P. Process Plants: A Handbook for Inherently Safer Design, Second Edition. CRC Press, 2010, 224 p.

6. Andersson H. A., van der Wijngaart W., Nilsson P. Valve-Less Diffuser Micropump for Microfluidic Analytical Systems. Proceedings of the $\mu$ TAS 2000 Symposium, Springer, 2000, pp. 95-98.

7. Stemme E., Stemme G. A Valveless Diffuser/Nozzle-Based Fluid Pump. Sensors and Actuators, 1993, no. 39, pp. 159-167.

8. Wada T., Takagi M., Shimizu A. Study on a Fluidic Pump with Vortex Diodes. Fluid Control and Measurement, Tokyo, Pergamon Press, 1986, pp. 421-426.

9. Takagi S., Saijo T. Study of a Piston Pump without Valves (1st Report, On a Pipe-capacity-system with a T-junction). Bull. JSME, 1983, no. 26, pp. 1366-1372. 
10. Takagi S., Takahashi K. Study of a Piston Pump without Valves (2st Report, On a Pipe-capacitysystem with a T-junction). Bull. JSME, 1985, vol. 28, no. 239, pp. 831-836.

11. Spiridonov E.K., Khabarova D.F. Raschetnaya model' i kharakteristiki besklapannogo porshnevogo nasosa odnostoronnego deystviya [Calculating Model and Characteristics Single Action Valveless Piston Pump]. Bulletin of the South Ural State University. Mechanical Engineering Industry, 2014, vol. 18, no. 3, pp. 5-12.

12. Spiridonov E.K., Khabarova D.F. Computational Model and Characteristics of a Double-Acting Valveless Piston Pump. Chemical and Petroleum Engineering, Springer, 2016, vol. 52, pp. 539-546.

13. Spiridonov E.K., Khabarova D.F. Printsipialnyye skhemy i kharakteristiki besklapannykh nasosov s vytesnitelem vozvratno-postupatel'nogo peremeshcheniya [Valveless pumps with displacer reciprocating movement. Schematic diagrams and operation processes]. Bulletin of the Samara Scientific Center of the Russian Academy of Sciences, 2016, vol. 18, no. 1(2), pp. 309-313.

14. Barmin I. V. Tekhnologicheskiye obekty nazemnoy infrastruktury raketno-kosmicheskoy tekhniki [Technological objects of ground-based infrastructure of rocket-and-space engineering Engineering manual. Book 1]. Moscow: Poligrafiks RPK, 2005, 416 p.

15. Barmin I. V. Tekhnologicheskiye ob'ekty nazemnoy infrastruktury raketno-kosmicheskoy tekhniki [Technological objects of ground-based infrastructure of rocket-and-space engineering. Engineering manual. Book 2]. Moscow: Poligrafiks RPK, 2006, 376 p.

\section{Об авторах}

Хабарова Дарья Федоровна (Челябинск, Россия) - старший преподаватель кафедры «Гидравлика и гидропневмосистемы» Южно-Уральского государственного университета (НИУ) (454080, г. Челябинск, пр. Ленина, д. 76, e-mail: khabarovadf@susu.ru).

Спиридонов Евгений Константинович (Челябинск, Россия) - доктор технических наук, профессор, заведующий кафедрой «Гидравлика и гидропневмосистемы» Южно-Уральского государственного университета (НИУ) (454080, г. Челябинск, пр. Ленина, д. 76, e-mail: spiridonovek@ susu.ru).

\section{About the authors}

Darya F. Khabarova (Chelyabinsk, Russian Federation) - Senior Lecturer, Department of Hydraulics and Hydraulic Systems, South Ural State University (National Research University) (76, Lenin av., Chelyabinsk, 454080, Russian Federation, e-mail: khabarovadf@susu.ru).

Evgeniy K. Spiridonov (Chelyabinsk, Russian Federation) - Doctor of Technical Science, Professor, Head of Department, of Hydraulics and Hydraulic Systems, South Ural State University (National Research University) (76, Lenin av., Chelyabinsk, 454080, Russian Federation, e-mail: spiridonovek@ @usu.ru). 